\title{
Proposed testing of kelea activated drinking water in zika virus infected pregnant women
}

\begin{abstract}
The current outbreak of Zika virus infections is particularly alarming because infection during pregnancy can lead to severe brain damage in the developing fetus. There are currently no anti-viral therapies being provided to Zika virus infected pregnant women. Methods are available to enhance the alternative cellular energy (ACE) pathway as a non-immunological anti-virus defense mechanism. Benefits of these therapies have been described in patients infected with herpes simplex virus (HSV), herpes zoster virus (HZV), human papillomaviruses (HPV) and human immunodeficiency virus (HIV). ACE pathway based therapies are particularly important in suppressing infections with stealth adapted viruses, which are not effectively recognized by the cellular immune system. As proposed elsewhere, co-infection with stealth adapted viruses may help explain the transplacental passage of the Zika virus into fetal tissues. Drinking water that has been activated with KELEA (kinetic energy limiting electrostatic attraction) can potentially provide a safe and effective means of enhancing the ACE pathway. Clinical trials using this and other approaches to stimulate the ACE pathway should be evaluated in Zika virus infected patients, including pregnant women. Zika virus infected mothers with brain damaged infants should also be tested for co-infection with stealth adapted viruses.
\end{abstract}

Keywords: Zika virus, Alternative cellular energy (ACE) pathway, Activated water KELEA, Kinetic energy limiting electrostatic attraction, Microcephaly, Autism, Stealth adapted viruses, SCMV, Enercel, HANSI, Lidocaine
Volume 3 Issue 5 - 2016

\author{
W John Martin \\ Institute of Progressive Medicine, USA
}

Correspondence: W John Martin, Institute of Progressive Medicine, 1634 Spruce Street, South Pasadena CA 91030, USA Tel (626) 6I6-2868 Email wjohmartin@ccid.org

Received: August 09, 2016 | Published: August 09, 2016
Abbreviations:ACE, Alternative Cellular Energy; HIV, Human Immunodeficiency Virus; HSV, Herpes Simplex Virus; HZV, Herpes Zoster Virus; HPV, Human Papillomaviruses; KELEA, Kinetic Energy Limiting Electrostatic Attraction; SCMV, African Green Monkey Simian Cytomegalovirus; CPE, Cytopathic Effect; CTL, Cytotoxic T Lymphocytes; CAM, Complementary and Alternative Medicine; PCR, Polymerase Chain Reaction

\section{Introduction}

Zika virus infections during pregnancy appear to differ from related dengue and yellow fever virus infections in the propensity of the Zika virus to infect the fetus and induce congenital birth defects. ${ }^{1,2}$ The developing brain is particularly susceptible to Zika virus induced damage, with first trimester infections not-uncommonly leading to microcephaly. ${ }^{3,4}$ Less severe forms of brain damage are also anticipated in many of the infected infants with normal head size. ${ }^{5}$ The Zika virus was initially described in $1947,6,7$ yet its association with microcephaly was only reported in $2015 .{ }^{8,9}$ It remains uncertain whether prior cases of microcephaly were simply not recognized as occurring in infants of Zika virus infected mothers or if the virus has newly acquired pathogenicity for the fetus. One possible explanation for the altered biological behavior of the Zika virus is that susceptible patients are co-infected with a virus that can render the placenta susceptible to the passage of Zika virus into the fetus. ${ }^{10}$

\section{Stealth adapted viruses and the alternative cellular energy (ace) pathway}

Stealth adapted viruses are derivative viruses, which have lost or mutated the relatively few major antigens normally targeted by the cellular immune system. ${ }^{11,12}$ These viruses, therefore, do not evoke the typical inflammatory response characteristic of infections with the conventional viruses from which the stealth adapted variants are derived. Stealth adapted viruses have been cultured from patients with various neuropsychiatric illnesses, including the chronic fatigue syndrome (CFS), ${ }^{13}$ bipolar psychosis ${ }^{14}$ and dementia. ${ }^{15}$ They have been cultured from children with autism ${ }^{16}$ and related illnesses ${ }^{17}$ and from several mothers of autistic children.

In spite of the absence of inflammation, stealth adapted virus infected patients do not exhibit an unrelenting progressive illness. Similarly, cats inoculated with stealth adapted viruses clinically recover from an acute non-inflammatory illness. ${ }^{18}$ A major inference from these and related studies is that the body is not solely dependent upon the cellular immune system as the major anti-virus defense mechanism. Indeed, as discussed below both stealth adapted and conventional viruses can be suppressed via an alternative cellular energy (ACE) pathway.

\section{KELEA (Kinetic Energy Limiting Electrostatic Attraction)}

The ACE pathway is an energy source that is distinct from cellular energy obtained from the metabolism of food. ${ }^{19-22}$ It involves a dynamic (kinetic) activity of the cellular fluids that is achieved by the absorption of an environmental force termed KELEA (kinetic energy limiting electrostatic attraction). The fundamental role of this force may be to prevent the fusion and possible annihilation of electrostatically attracted opposite electrical changes. KELEA is seemingly reversibly attracted to separated electrical charges. It may accompany electromagnetic radiation and possibly also cosmic rays. ${ }^{23}$ KELEA can diminish the electrostatic hydrogen bonding between fluid molecules, including water, ${ }^{24}$ ethano ${ }^{25}$ and gasoline ${ }^{26}$ KELEA can be assayed by its capacity to increase the volatility of these fluids in closed but not completely sealed containers, as measured by reductions in weight. KELEA can also influenced measured weight in other ways that are currently being investigated. ${ }^{27}$ 


\section{ACE pathway based anti-viral therapies}

The efficacy of various modalities of Complementary and Alternative Medicine (CAM) can be explained through their ability to bring KELEA into the body and, thereby, enhance the body's ACE pathway. ${ }^{28}$ Clinical efficacy of ACE pathway activation using a phototherapy procedure has been shown with HSV, HZV and HPV. ${ }^{29,30}$ A related phototherapy protocol was highly effective in treating several children with autism. ${ }^{31}$ More direct activation of the ACE pathway has been achieved using a "homeopathic" formulation termed Enercel. Its formulation is based on a similar product termed HANSI (homeopathic activator of the natural system immune). Enercel and HANSI are typically administered by injection, although they can be used as a nasal spray and as an inhalant. Enercel is effective in suppressing the reactivation of the cytopathic effect (CPE) of stealth adapted viruses (unpublished). It is clinically effective in a wide range of infectious clinical illnesses, ${ }^{32}$ including herpes infections, children with rotavirus induced diarrhea ${ }^{33}$ and AIDS. ${ }^{34}$

Although reportedly homeopathic, tested batches of injectable Enercel and HANSI contain undisclosed, yet detectable levels of Lidocaine (xylocaine). The related chemical procaine is included in the general health promoting and rejuvenation formulation called Gerovital. ${ }^{35}$ Review of these and many additional compounds with stated broad based therapeutic benefits, indicate that they have a common characteristic of being electrically dipolar. Examples include humic/fulvic acids, zeolites, magnesium oxide, moringa powder, cocoa, various herbal tinctures and many others. Dipolar compounds can seemingly attract KELEA and some can transfer the energy into nearby fluids, possibly in an oscillatory manner. ${ }^{36,37}$ Once the fluid is activated, insoluble compounds can be removed by decanting and soluble compounds removed by repeated dilutions, as in homeopathy, or by zero-residue. KELEA activated water is characterized by several physical properties, including lower surface tension and increased volatility, consistent with the aforementioned reduction in the strength of intermolecular hydrogen bonding. Many healing devices operate by generating fluctuating electrical and/or electromagnetic energies. Examples include the Beam Ray of Royal Raymond Rife; Multiwave Oscillator of Georges Lakhovsky; and the Papimi machine of Panos Papas. ${ }^{38}$ Interacting light fields similar to those in the Energy Enhancement System (EESystem) of Sandra Rose Michael and bidirectional direct current as suggested by Nikola Tesla, have both been shown shown to readily activate water. KELEA activated water can also activate water placed within close proximity for an extended period. ${ }^{39,40}$

\section{Proposed Anti-Zika virus studies}

Enhancing the ACE pathway using KELEA activated drinking water may provide an effective mechanism of reducing fetal brain damage in Zika virus infected pregnant women. ${ }^{41}$ A suggested protocol is to consume approximately one gallon per week of water that has been externally activated. Using external activation removes any concerns with the possible toxicity of added components, even those these components can be subsequently removed. Methods can be expanded to achieve KELEA activation of entire municipal supplies of drinking water. Bathing in KELEA activated water may also be incorporated into testing protocols. More highly activated water and other KELEA generating devices can be tested for their anti-virus activity in Zika virus cultures.

An added potential benefit of consuming KELEA activated water is that an enhanced ACE pathway may additionally act as a mosquito repellent. It may do so by adding to the negative ionization surrounding the body, which mosquitoes tend to avoid. Empirical clinical data are providing support for this premise. Furthermore, farmers have noted that crops fed with KELEA attracting compounds can be distinguished from regular crops by having less hovering insects (unpublished observations).

The second major research undertaking should be testing Zika virus infected women, who have delivered brain damaged infants, for evidence of their being infected with stealth adapted viruses. As noted above, these are cytopathic viruses in which there is either deletion or mutation of the relatively few viral genes, which prior to stealth adaptation, would have coded antigens targeted by cytotoxic $\mathrm{T}$ lymphocytes (CTL). Stealth adaptation is viewed as a generic process, which can occur with all conventional viruses. The brain is particularly susceptible to symptomatic illness caused by stealth adapted viruses. Evidence can be sought for cognitive, mood and/or sleep disorders in women prior to their becoming pregnant and Zika virus infected.

The diverse origins of stealth adapted viruses limit the usefulness of molecular screening assays, such as the polymerase chain reaction (PCR). Furthermore, the viruses can undergo additional molecular changes because of genetic instability ${ }^{42}$ and also yield unexpected results from the incorporation of additional genes of both cellular ${ }^{43}$ and bacteria ${ }^{44}$ origins. The most reliable screening method for stealth adapted viruses is virus cultures.

Detailed protocols have been provided to Public Health officials concerning the culture of stealth adapted viruses. Essentially the only adjustments to routine virus cultures are i) use of frozen-thawed mononuclear cells; ii) preference for serum-free medium; and iii) frequent re-feeding of cultures. This reduces the level of accumulating ACE pigments, which can otherwise suppress of the virus induced CPE. The CPE is broadly characterized by the formation of foamy, vacuolated cells, sometimes with marked syncytia. ${ }^{45}$ The formation of ACE pigments can be seen using ultraviolet fluorescence, especially when using neutral red or acridine orange dyes. ${ }^{45}$ Extracellular lipids, especially in the form of long troughs, can develop in long term cultures. ${ }^{45}$ Extracellular DNA can also be cloned and sequenced allowing for the better use of PCR assays to molecularly characterize the likely origin of different viruses. ${ }^{42}$ Of particular interest is the reported finding that certain stealth adapted are unequivocally derived from African green monkey simian cytomegalovirus (SCMV). ${ }^{12,46}$

Incorporating stealth adapted virus testing in the proposed KELEA activated water consumption studies may well expedite efforts to reduce the transplacental transmission of stealth adapted viruses; the proposed cause of autism and of related illnesses in children and many neuropsychiatric illnesses in adults.

\section{Conclusion}

Public Health authorities have been reluctant to acknowledge the existence of stealth adapted viruses, especially since some were unequivocally derived from SCMV. ${ }^{47}$ It will be unfortunate if this disregard delays consideration of stealth adapted viruses as cofactors in Zika virus pathogenicity. Indeed, stealth adapted viruses may well be a co-factor in HIV infections and potentially also in the initial occurrence of HIV in polio vaccine inoculated chimpanzees. ${ }^{47}$ A major outcome of studies on stealth adapted viruses has been the identification of the ACE pathway as a non-immunological anti-virus defense mechanism. The possible benefits of consuming KELEA activated water in Zika virus infected pregnant women should be evaluated. Zika infected mothers of severely brain damaged infants should also be tested for stealth adapted viruses. 


\section{Acknowledgements}

The Institute of Progressive Medicine is a component of MI Hope Inc., a non-profit public charity.

\section{Conflicts of interest}

None.

\section{References}

1. Chan JF, Choi GK, Yip CC et al. Zika fever and congenital Zika syndrome: An unexpected emerging arboviral disease. J Infect. 2016;72(5):507-524.

2. Boeuf P, Drummer HE, Richards JS The global threat of Zika virus to pregnancy: epidemiology, clinical perspectives, mechanisms, and impact. BMC Medicine. 2016;14(1):112.

3. Mlakar J, Korva M, Tul N et al. Zika virus associated with microcephaly. N Engl J Med. 2016;374(10):951-958.

4. Valentine G, Marquez L, Pammi M Zika Virus-Associated Microcephaly and Eye Lesions in the Newborn. J Pediatric Infect Dis Soc (in press). 2016

5. Oliveira Melo AS, Malinger G, Ximenes R et al. Zika virus intrauterine infection causes fetal brain abnormality and microcephaly: tip of the iceberg? Ultrasound Obstet Gynecol. 2016;47(1):6-7.

6. Weaver SC, Costa F, Garcia-Blanco MA et al. Zika virus: History, emergence, biology, and prospects for control. Antiviral Res. 2016;130:69-80.

7. Zanluca C, Dos Santos CN Zika virus-an overview. Microbes Infect. 2016;18(5):295-301.

8. European Centre for Disease Prevention and Control Rapid risk assessment: Zika virus epidemic in the Americas: potential association with microcephaly and Guillain-Barré syndrome. Stockholm, Sweden. 2015

9. Schuler-Faccini L, Ribeiro EM, Feitosa IM et al. Possible association between Zika virus infection and microcephaly-Brazil, 2015. MMWR Morbidity Mortality Weekly Report. 2016;65(3):59-62.

10. Martin WJ Co-infection with stealth adapted viruses may explain Zika virus associated microcephaly. Journal Human Virology \& Retrovirology. 2016;3(1):00080.

11. Martin WJ Stealth Adapted Viruses; Alternative Cellular Energy (ACE) \& KELEA Activated Water. Author House, Blomington, USA, pp. 2014;332.

12. Martin WJ Stealth adaptation of viruses: Review and updated molecular analysis on a stealth adapted African green monkey simian cytomegalovirus (SCMV). Journal of Human Virology \& Retrovirology. 2014;1(4):00020.

13. Martin WJ, Zeng LC, Ahmed K et al. Cytomegalovirus related sequences in an atypical cytopathic virus repeatedly isolated from a patient with the chronic fatigue syndrome. Am J Pathol. 1994;145(2):440-451.

14. Martin WJ Simian cytomegalovirus related stealth virus isolated from the cerebrospinal fluid of a patient with bipolar psychosis and acute encephalopathy. Pathobiology. 1996;64(2):64-66.

15. Martin WJ Severe stealth virus encephalopathy following chronic fatigue syndrome like illness: Clinical and histopathological features. Pathobiology. 1996;64(1):1-8.

16. Martin WJ Stealth virus isolated from an autistic child. J Autism Dev Disord. 1995;25(2):223-224.

17. Martin WJ, Anderson D Stealth virus epidemic in the Mohave Valley: Severe vacuolating encephalopathy in a child presenting with a behavioral disorder. Exp Mol Pathol. 1999;66(1):19-30.
18. Martin WJ, Glass RT Acute encephalopathy induced in cats with a stealth virus isolated from a patient with chronic fatigue syndrome. Pathobiology. 1995;63(3):115-118.

19. Martin WJ Stealth virus culture pigments: A potential source of cellular energy. Exp Mol Pathol. 2003;74(3):210-223.

20. Martin WJ Progressive medicine. Exp Mol Pathol. 2005;78(3):218-220.

21. Martin WJ Etheric biology. Exp Mol Pathol. 2005;78(3):221-227.

22. Martin WJ Deconstructing medicine. The alternative cellular energy pathway. British Journal of Medicine \& Medical Research. 2016;11(8):1-6.

23. Martin WJ KELEA, Cosmic rays, cloud formation and electromagnetic radiation: Electropollution as a possible explanation for climate change. Atmospheric and Climate Sciences. 2016;6:174-179.

24. Martin WJ KELEA: A natural energy that seemingly reduces intermolecular hydrogen bonding in water and other liquids. Open Journal of Biophysics. 2015;5:69-79.

25. Martin WJ KELEA Activation of water and other fluids for health, agriculture and industry. JWARP. 2015;7(16):1331-1344.

26. Martin WJ. KELEA (kinetic energy limiting electrostatic attraction) can markedly improve the performance of gasoline and diesel fuels in power generation and in transportation. Journal of Transportation Technologies. 2016;6:148-154.

27. Martin WJ. KELEA (kinetic energy limiting electrostatic attraction) may add to the measured weight of an object. JMP. 2016;7(6):461-472.

28. Martin WJ. Alternative cellular energy as a unifying concept in complementary alternative medicine. International Journal Complementary \& Alternative Medicine. 2015;1(4):00022.

29. Martin WJ, Stoneburner J. Symptomatic relief of herpetic skin lesions utilizing an energy based approach to healing. Exp Mol Pathol. 2005;78(2):131-134.

30. Martin WJ, Stoneburner J. Alternative cellular energy (ACE) pathway activation as the mode of action of neutral red dye phototherapy of human viruses. Journal of Human Virology \& Retrovirology. 2014;1(4):00019.

31. Martin WJ. Alternative cellular energy (ACE) pathway activation as natural therapy for autism. In Stealth Adapted Viruses; Alternative Cellular Energy (ACE) \& KELEA Activated Water. Author House, Blomington IN, USA. 2014;p.87-102.

32. Martin WJ, Laurent D. Homeopathy as a misnomer for activation of the alternative cellular energy pathway: Evidence for the therapeutic benefits of Enercel in a diverse range of clinical illnesses. International Journal Complementary \& Alternative Medicine. 2015;2(1):00045.

33. Martin WJ. Alternative cellular energy based therapy of childhood diarrhea. In Stealth Adapted Viruses; Alternative Cellular Energy (ACE) \& KELEA Activated Water. Author House, Blomington IN, USA. 2014;pp.103-114.

34. Dubrov V, Dubrova T, Christner D, et al. Alternative cellular energy based therapy using Enercel ${ }^{\circledR}$ in advanced AIDS patients co-infected with tuberculosis and treated in Chernigov, Ukraine. Journal of Human Virology \& Retrovirology. 2015;2(6):00061.

35. Gang Xu, Zhaochun Cao, Mina Shariff, et al. Effects of G.H.3. on mental symptoms and health-related quality of life among older adults: results of a three-month follow-up study in Shanghai, China. Nutrition Journal. 2016;15: 9.

36. Martin WJ. Alternative cellular energy pathway therapy using KELEA activated water. International Journal Complementary \& Alternative Medicine. 2015;2(2):00051.

37. Martin WJ. Preparing and using KELEA activated water to enhance the alternative cellular energy (ACE) pathway in the therapy of multiple illnesses. International Journal Complementary \& Alternative Medicine. 2016;3(1):00059. 
38. Martin WJ. Interacting light paths attract KELEA (kinetic energy limiting electrostatic attraction) and can lead to the activation of water Open Journal of Biophysics. 2015;5:115-121.

39. Martin WJ. Interactive electric fields can attract KELEA (kinetic energy limiting electrostatic attraction) and can lead to the activation of water. International Journal Complementary \& Alternative Medicine. 2015;1(6):00034.

40. Martin WJ. Cancer as an insufficiency of cellular energy (ICE): Therapeutic approaches based on enhancing the alternative cellular energy (ACE) pathway. International Journal Complementary \& Alternative Medicine. 2016;3(3):00074.

41. Martin WJ. Enhancing the alternative cellular energy (ACE) pathway in the prevention of Zika virus induced illness. Journal of Human Virology \& Retrovirology. 2016;3(4):00097.

42. Martin WJ. Genetic instability and fragmentation of a stealth viral genome. Pathobiology. 1996;64(1):9-17.
43. Martin WJ. Cellular sequences in stealth viruses. Pathobiology. 1998;66(2):53-58

44. Martin WJ. Bacteria related sequences in a simian cytomegalovirusderived stealth virus culture. Exp Mol Pathol. 1999;66(1):8-14.

45. Martin WJ. The alternative cellular energy (ACE) pathway in the repair of the cytopathic effect (CPE) caused by stealth adapted viruses: In vitro and in vivo evidence supporting a new therapeutic paradigm. In Stealth Adapted Viruses; Alternative Cellular Energy (ACE) \& KELEA Activated Water. Author House, Blomington IN. 2014;31-70.

46. Martin WJ, Ahmed KN, Zeng LC, et al. African green monkey origin of the atypical cytopathic stealth virus isolated from a patient with chronic fatigue syndrome. Clin Diagn Virol. 1995;4(1):93-103.

47. Martin WJ. Chimpanzees inoculated with cytomegalovirus contaminated polio vaccines may explain origin of HIV-1. Journal of Human Virology \& Retrovirology. 2015;2(2):00035. 